\title{
Study on the Position and Function of Functions in Mathematics
}

\author{
Zhifeng Yu \\ Nanchang Institute of Science \& Technology
}

\begin{abstract}
Keywords: Function; Higher mathematics; Role; The function idea
\end{abstract}
\begin{abstract}
Function is a kind of important method to solve the problem in mathematical analysis. The essence of function method is a kind of mathematical thinking method to solve the problem by using function knowledge to construct the function equation for problem solution based on the analysis and understanding of the problem met in actual life. The construction of a proper auxiliary function in processing and solving the problem will make the solution very simple. Therefore, in order to make students grasp related content better of the function, mathematics teachers should constantly strengthen the study of education theory, timely and accurately grasp the minds of students, improve teaching, making teaching become a feast for the eyes and creative activities, guiding students to consciously eliminate psychological barriers of mathematics learning, heading for higher and further target. This paper article states the role and status of function in the mathematics teaching from the 3 aspects of function development, function thinking and function application.
\end{abstract}

\section{Introduction}

Functions have several significant developments. Mathematics history shows that the function concept has experienced a long and tortuous process from emergence to perfection. This not only because the function concept system is complicated and involves many factors, the more important is the mathematical thinking way also has an important turning point accompanied by the development function concept: thinking from static to dynamic, from discrete to continuous, from operation to the relationship, realizing the organic combination of numbers and forms, and the flexible conversion between symbolic language and graph and table language. In the function study, thinking exceeds the limits of formal logic, and enters the dialectical logic thinking. Compared with the constant mathematics, function concept is more abstract and more formalized.

Function is the important content in mathematics, and its knowledge runs through the whole process of mathematics teaching. Function exists in each knowledge domain, and it exists everywhere. There is no mathematics without function; function is the core of mathematics and the basis of subsequent development of mathematics. At the same time, in the natural sciences such as physics, chemistry, it has been widely used, and function is often used as the basic modeling tool in solving the practical problems in production and living. Function, therefore, the study of function is very important, which should be attached great attention.

\section{Function Idea}

Function idea is embodied at: one thinking strategy in solving the problem of "mathematical model".

In particular, function describes the relationship of numbers in nature, and function idea performs study through raising the mathematical characteristics of questions and setting up the mathematic model of function relationship type. It embodies the dialectical materialist idea of "connection and change". Generally, function idea is to construct function (that is, "regulation idea") and solve problems by using the properties of function (known + unknown + regulation idea). The natures often used include: monotonicity of $f(x)$ and $x$, parity, periodicity, the maximum and the minimum value, image transformation and so on, so that we are required to master the specific properties of linear function, quadratic function, power function, exponential function, logarithm function and trigonometric function. 
In the problem solving, it is the key to use function idea to be good at mining the hidden conditions and constructing the function analytic expression, as well as using the natures of functions. Only if the observation, analysis and judgment on the given issue are deep, full and comprehensive, the related link can be created and the function prototype can be constructed. In addition, the equation, inequation problem, and some algebraic problems can also be converted into the relevant problem of function, namely to solve the non-function problems with function idea.

For many mathematics problems, it seems to be more difficult, but they can be solved using the function idea to transform them into function problem, how to use the function idea to solve mathematical problems and reflect the essence of function idea in the process of problem solving.

\section{Application of Function in Mathematics}

Figure out Set Problem with Function Idea: Eg.1 if $A=\{x \mid-2 \leq x \leq a\}, \quad B=\{y \mid y=2 x+3 \quad, \quad x \in$ A $\}, \quad C=\left\{z \mid z=x^{2}, \quad x \in A\right\}$ and $C \mid B$, figure out the value range of $a$.

Idea: image association, defining number with images, namely associate the image from the function in condition $\mathrm{B}, \mathrm{C}$, and then the inclusive relationship between sets is visualized and the corresponding algebra relationship is determined to avoid classified discussion for a. As each function corresponds with an image, the function problems carried by sets often use the function images to reflect the relationship between sets to solve the problems.

Figure out Unequal Problems with Function Idea: Eg.2 Let inequation
$\frac{1}{\mathrm{n}+1}+\frac{1}{\mathrm{n}+2}+\cdots \frac{1}{3 \mathrm{n}+1}>2 \mathrm{a}-5$, and $\mathrm{n} \in \mathrm{N}^{*}$, figure out the maximum value of natural number $\mathrm{a}$.

Analysis: construct a function, and let

$$
\mathrm{f}(n)=\frac{1}{n+1}+\frac{1}{n+2} \cdots \frac{1}{3 n+1}\left(n \in \mathrm{N}^{*}\right)
$$

If

$f(n+1)-f(n)=\frac{1}{3 n+2}+\frac{1}{3 n+3}+\frac{1}{3 n+4}-\frac{1}{n+1}$

$=\frac{1}{3 n+2}+\frac{1}{3 n+4}-\frac{2}{3 n+3}$

$=\frac{2}{3(n+1)(3 n+2)(3 n+4)}>0$

$\mathrm{f}(\mathrm{n}+1)>\mathrm{f}(\mathrm{n})\left(\mathrm{n} \in \mathrm{N}^{*}\right)$ can be got, that is $\mathrm{f}(\mathrm{n})>\mathrm{f}(\mathrm{n}-1)>\ldots>\mathrm{f}(2)>\mathrm{f}(1)$, therefore, the minimum value of $f(n)$ is $f(1)=\frac{13}{12}$. And the sufficient condition for all the $n \in N^{*}, f(n)>2 a-5, \frac{13}{12}>2 a-5$, that is a $<\frac{73}{24}$. Thus, the maximum value for natural number a is 3 .

Solve the Algebra Problems with Function Idea. Eg 3. The known real number Q and p can comply with these two equations $\alpha^{3}-\alpha^{2}+5 \alpha=1, \beta^{3}-3 \beta^{3}+5 \beta=5$, try to figure out the value of $\alpha+\beta$.

Analysis: transform the equation into $\alpha^{3}-\alpha^{2}+5 \alpha=1,(\beta-1)^{3}+2(\beta-1)=2$. From the features of the left formula, function $\mathrm{f}(\mathrm{x})=\mathrm{x}^{3}+2 \mathrm{x}$ can be introduced. Then the question is transformed into " $f(\alpha-1)=-2, f(\beta-1)=2$ is known, figure out the value of $\alpha+\beta$ ". As function monotony is the dependency between the independent variable and function value. Therefore, this question can be solved with function monotony.

Analysis: this question constructs functions with the help of function monotony.

Solve Logarithm Question with Function Idea. Eg 4. For the real number $\mathrm{x}$ with $0 \leq \mathrm{p} \leq 4$, figure out the value range of $x$ that making inequation $\lg 2 x+p \lg x>4 \lg x+p-3$ established.

Analysis: to prove $\lg 2 x+p \lg x>4 \lg x+p-3,(\lg x-1) p+\lg 2 x-4 \lg x+3>0$ should be proved firstly. 
Introduce function $f(p)=(\lg x-1) p+\lg 2 x-4 \lg x-3$, the question is transformed into figuring out the value range of $x$ when $f(p)=(\lg x-1) p+\lg 2 x-4 \lg x-3$ and $f(p)>0$. As $f(p)$ and $p$ have linear relation, the inequation cannot be established only if $f(0)>0$ and $f(4)>0$.

In function applications, there are three levels: one is the experience of process and method to establish a function model; Secondly, to solve practical problems with a given model; thirdly, to establish "certainty" function model and solve the problem according to the data fitting function.

\section{The Function Position and Role}

In addition to reflecting an essential attribute on the dependency relationship between variables, the concept of function in higher mathematics also has a variety of other properties. Function is closely linked with the concepts of limit, continuity, derivative, differential, integral and differential equations, etc. For overall understanding of the function concept, its various internal attributes and internal and external relationships should be investigated and grasped comprehensively and systematically. To achieve this goal, it is necessary to methodize and systematize the whole knowledge of function to form the relatively independent knowledge structure.

Function is one of the important basic concepts of mathematics. The mathematics analysis needing further study, including the limit theory, differential calculus, integral calculus, differential equation and functional analysis and other mathematics foundation courses opened by colleges and universities, all take function as the basic concept and the research object. Other subjects such as physics, also take the basic knowledge of function as a tool to research solve problems. Function teaching content contains an extremely rich dialectical thought, so it is good material for students to accept the dialectical materialism education. Moreover, the way of thinking of function is also widely penetrated into the whole process of middle school mathematics and university mathematics and other subjects.

Great mathematicians Klein put forward such a resounding slogan "think with function" in leading the modern mathematics education reform movement. This also demonstrates the practical significance to solve the problem and explore the mathematical world law of development with function idea. Actually, from beginning to end, the function runs through the higher mathematics teaching material, it often can have very good effect with grasping function idea well and solving some problems in higher mathematics.

\section{Conclusion}

This paper states the development of function and its importance. According to the function idea described in this paper, the application of function in solving mathematical problem, and the role and status of function from function application, from this paper, the students cannot only realize, accurate knowledge of function can provide powerful basis for practice to make scientific decision and when the precise function knowledge is used in practice, corresponding function expressions can be selected based on specific questions, and make appropriate adjustments according to the development process. From this paper, the contents of function can be better grasped by the students, who can also have a more profound impression for function, improving the interest of function learning. Therefore, it will have important significance in theoretical study and practical application with fully understanding the concept of function and grasping the method of function.

\section{References}

[1] Hersey C D, Way R B. Computer Science and Applied Mathematics: A SERIES OF MONOGRAPHS AND TEXTBOOKS - Symbolic Logic and Mechanical Theorem Proving[D]. US, 1956.

[2] Marsden J E, Ratiu T S. Texts in Applied Mathematics[M]// Studies in applied mathematics. The MIT Press, 1969:B33. 
[3] Ng C K Y, Britta W, Roger A, et al. Computer Science and Applied Mathematics: A SERIES OF MONOGRAPHS AND TEXTBOOKS- Constrained Optimization and Lagrange Multiplier Methods [J]. Cancer Research, 2014, 74(11):ibc1-ibc2. Press A. Advances in applied mathematics. [M]. Academic Press. 1980.

[4] Von Mises R. Applied Mathematics. (Scientific Books: Quarterly of Applied Mathematics)[J]. Science, 1944, 99(99):81-82.

[5] Teague J. Computer Science and Applied Mathematics: A SERIES OF MONOGRAPHS AND TEXTBOOKS - Computer Arithmetic in Theory and Practice [J]. University of Queensland Australia, 2000:155-163.

[6] Colyvan M. The Miracle of Applied Mathematics [J]. Synthese, 2001, 127(3):265-278.

[7] Olshausen B A, Deweese M R. Applied mathematics: The statistics of style.[J]. Nature, 2010, 463(7284):1027-8.

[8] Simos T E, Tsitouras C, Psihoyios G. NUMERICAL ANALYSIS AND APPLIED MATHEMATICS: International Conference on Numerical Analysis and Applied Mathematics 2009: Volume 1 and Volume 2[J]. 2008.

[9] Ryan J. IIS-Hypergraphs : SIAM Journal on Discrete Mathematics: Vol. 9, No. 4 (Society for Industrial and Applied Mathematics)[J]. Siam Journal on Discrete Mathematics, 2006.

[10]Jaeger J C, Starfield A M. An introduction to applied mathematics,[M]. Clarendon Press, 1974.

[11]Jesús Vigo-Aguiar, Bruce A. Wade. Recent advances in computational and applied mathematics in science and engineering [J]. International Journal of Computer Mathematics, 2009, 86(2):199-199. 